\title{
Multi-agent Self-organization and Reorganization to Adapt M2M Infrastructures
}

\author{
Gauthier Picard $^{\star \dagger} \quad$ C. Persson ${ }^{\star \ddagger} \quad$ O. Boissier ${ }^{\star \dagger} \quad$ F. Ramparany ${ }^{\ddagger}$
}

* MINES Saint-Étienne

$\dagger$ LaHC UMR CNRS 5516

¥ Orange Labs/COSY

September 24th, 2015
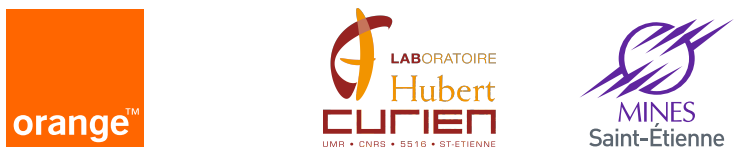


\section{Machine-†o-Machine (M2M)}

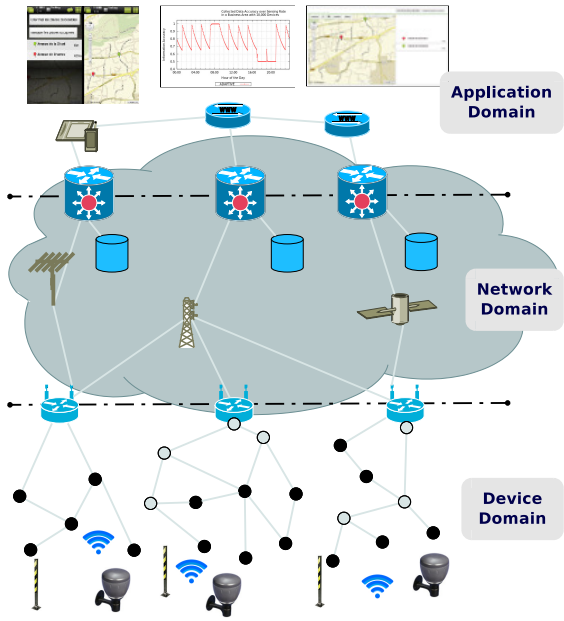

¿ Used by multiple applications 


\section{M2M Infrastructures}

- Vertical M2M Infrastructure

$\checkmark$ Control over Devices

$x$ Expensive Cost

$x$ Limited reuse between Apps

$x$ Small Scale Deployment

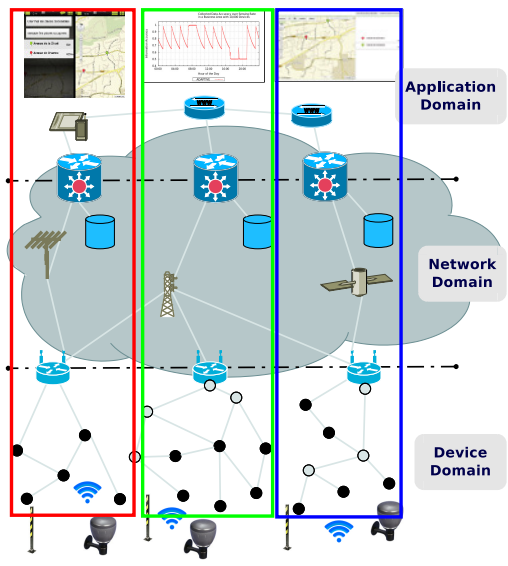




\section{M2M Infrastructures}

- Vertical M2M Infrastructure

$\checkmark$ Control over Devices

$x$ Expensive Cost

$\boldsymbol{x}$ Limited reuse between Apps

x Small Scale Deployment

- Multi-Vertical M2M Infrastructure

$\checkmark$ Control over Devices

$\checkmark$ Shared Infrastructure Cost

$\boldsymbol{x}$ Limited reuse between Apps

$x$ Medium Scale Deployment

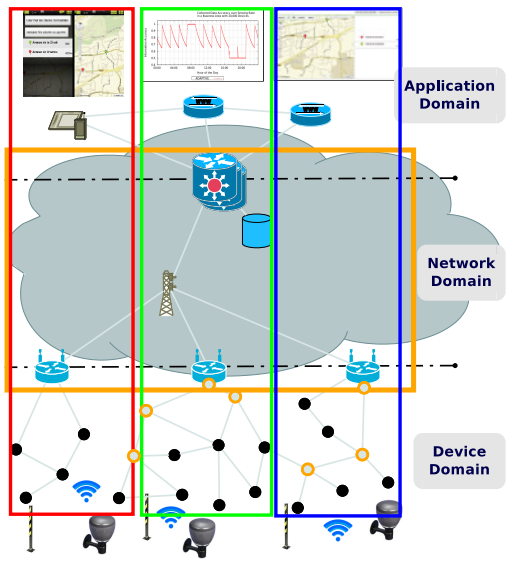




\section{M2M Infrastructures}

- Vertical M2M Infrastructure

$\checkmark$ Control over Devices

$x$ Expensive Cost

$x$ Limited reuse between Apps

x Small Scale Deployment

- Multi-Vertical M2M Infrastructure
$\checkmark$ Control over Devices
$\checkmark$ Shared Infrastructure Cost
$\boldsymbol{x}$ Limited reuse between Apps
$x$ Medium Scale Deployment

- Horizontal M2M Infrastructure

$x$ Less Control over Devices

$\checkmark$ Shared Infrastructure Cost

$\checkmark$ Reuse of Devices between Apps

$\checkmark$ Large Scale Deployment

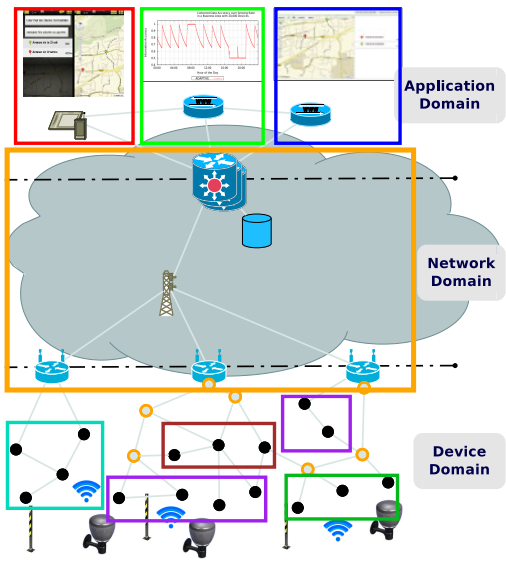




\section{M2M Infrastructures}

- Vertical M2M Infrastructure

$\checkmark$ Control over Devices

$x$ Expensive Cost

$x$ Limited reuse between Apps

x Small Scale Deployment

- Multi-Vertical M2M Infrastructure
$\checkmark$ Control over Devices
$\checkmark$ Shared Infrastructure Cost
$\boldsymbol{x}$ Limited reuse between Apps
x Medium Scale Deployment

- Horizontal M2M Infrastructure

$x$ Less Control over Devices

$\checkmark$ Shared Infrastructure Cost

$\checkmark$ Reuse of Devices between Apps

$\checkmark$ Large Scale Deployment

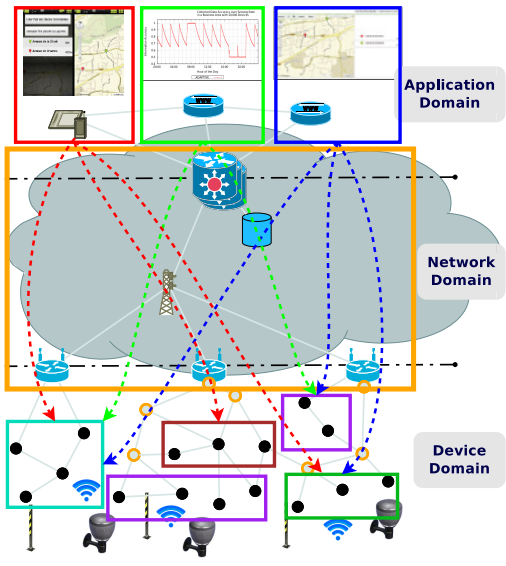




\section{Problem Statement}

- How to balance Vertical and Horizontal Benefits?

- How to control the horizontal M2M infrastructure to guarantee Devices Constraints and Application Requirements?

- How to adapt the M2M infrastructure Dynamics and Scale?

- Definition of a Machine-to-Machine specific Governance

- Automated Control and Adaptation Governance processes

- Decentralized and Distributed Governance processes 


\section{Outline}

M2M, Governance and Adaptation

AGaMeMnON : an Adaptive Governance MAS for M2M

Self-reorganization in AGAMEMNON

Implementation and Evaluation

Conclusion 


\section{Governance Principles in ICT}

COBIT $\left(\mathrm{HBC}^{+}{ }^{07}\right), \mathrm{IBM}$ SOA Governance $(\mathrm{BCO})$

- 3 Governance Levels

- Strategy : Functional / Non-Functional Requirements Specification e.g. IT Goals, Requirements Planning

- Tactic : Intermediate Goals Definition e.g. Process Goals, Approach Definition

- Policy : Resource Usage Management e.g. Activity Goals, Model Enabling

- Governance Process

- Governance Refinement: Performance Analysis and Redefinition e.g. Measurement and Realignment, Measurement and Refinement 


\section{Governance Principles in M2M}

- 3 Governance Levels

- Strategy : Functional / Non-Functional Requirements Specification

- Horizontal : ETSI TC M2M - Functional Architecture

- Vertical: Service Level Agreement (SLA)

- Policy : Resource Usage Management

- Device Management Recommendations

- Device Management Protocols

- Platform's Device Abstraction

\section{- Governance Process}




\section{Governance Principles in M2M}

- 3 Governance Levels

- Tactic : Intermediate Goals Definition

- Governance Process

- Governance Adaptation : Performance Analysis and Redefinition 


\section{Governance Approaches in M2M}

Control and Adaptation Systems

Endogenous Control and Adaptation

Mix the Business Logic processes and the Governance processes

- Ad-Hoc Adaptative Algorithms

- Stochastic/Probabilistic Algorithms

- Swarm Intelligence

- Clustering Algorithm

- Governance Pros/Cons

- Requirements Definition:

Not Explicit, Less Dynamic, Procedural

- Control:

Bottom-Up, No Global State, No Guaranty

- Adaptation:

Fast Reactivity, Locally
Exogenous Control and Adaptation

Separate the Business Logic processes and the Governance processes

- Requirement-based Control Loop

- Middleware

- Autonomic Computing

- Multi-Agent Systems (MAS)

- Governance Pros/Cons

- Requirements Definition: Explicit, Dynamic, Declarative

- Control: Top-Down, Global State, Better Guaranty

- Adaptation: Less Reactive, Globally

$\Rightarrow$ Combined with Endogenous 


\section{Governance Approaches in M2M}

Control and Adaptation Systems

Endogenous Control and Adaptation

Mix the Business Logic processes and the Governance processes

- Ad-Hoc Adaptative Algorithms

- Stochastic/Probabilistic Algorithms

- Swarm Intelligence

- Clustering Algorithm

- Governance Pros/Cons

- Requirements Definition:

Not Explicit, Less Dynamic, Procedural

- Control:

Bottom-Up, No Global State, No Guaranty

- Adaptation:

Fast Reactivity, Locally
Exogenous Control and Adaptation

Separate the Business Logic processes and the Governance processes

- Requirement-based Control Loop

- Middleware

- Autonomic Computing

- Multi-Agent Systems (MAS)

- Governance Pros/Cons

- Requirements Definition: Explicit, Dynamic, Declarative

- Control: Top-Down, Global State, Better Guaranty

- Adaptation: Less Reactive, Globally

$\Rightarrow$ Combined with Endogenous 


\section{Multi-Agent Oriented Programming}

Organization

- Collective Behavior : Role-Social Goals-Norms

- Functional / Non-Functional Requirements

\section{Agent}

- Reactive and Proactive Processes (e.g. BDI)

- Requirement $\rightarrow$ Control Decisions

\section{Environment}

- First-class Abstraction : Resources, Communication

- External Business Entities Abstraction

Interaction

- Protocols to bind all MAS entities

Reorganization

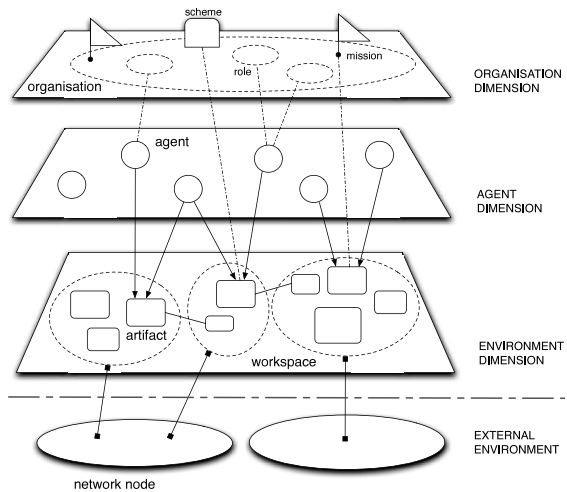

VOWELS dimensions (Dem95)

- Redefine the Organization Specification/Entity

- Top-Down / Bottom-Up 


\section{Multi-Agent Oriented Programming}

Organization .................. Strategy

- Collective Behavior : Role-Social Goals-Norms

- Functional / Non-Functional Requirements

Agent ........................ Tactic

- Reactive and Proactive Processes (e.g. BDI)

- Requirement $\rightarrow$ Control Decisions

Environment ................... Policy

- First-class Abstraction : Resources, Communication

- External Business Entities Abstraction

Interaction

- Protocols to bind all MAS entities

Reorganization ..............Adaptation

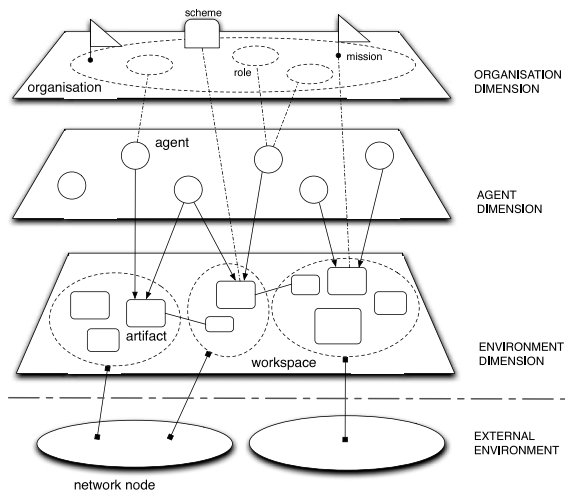

VOWELS dimensions (Dem95)

- Redefine the Organization Specification/Entity

- Top-Down / Bottom-Up 


\section{Outline}

\section{M2M, Governance and Adaptation}

AGaMeMnON : an Adaptive Governance MAS for M2M

\section{Self-reorganization in AGAMEMNON}

Implementation and Evaluation 


\section{Overview of the AGAMEMNON Framework}

- Organization-based Governance Strategy

Normative

MOISE (HBO2)

- Agent-based Governance Tactic Declarative

Jason (BHWO8)

- Artifact-based Governance Policy Resource Abstraction CArtAgO (ORVO8)

- Synergistic Integration JaCaMo (PBRH1O)

- MAS Adaptation Governance Refinement cycle

- Top-Down Enactment

- Horizontal Adaptation

- Bottom-Up Reorganization

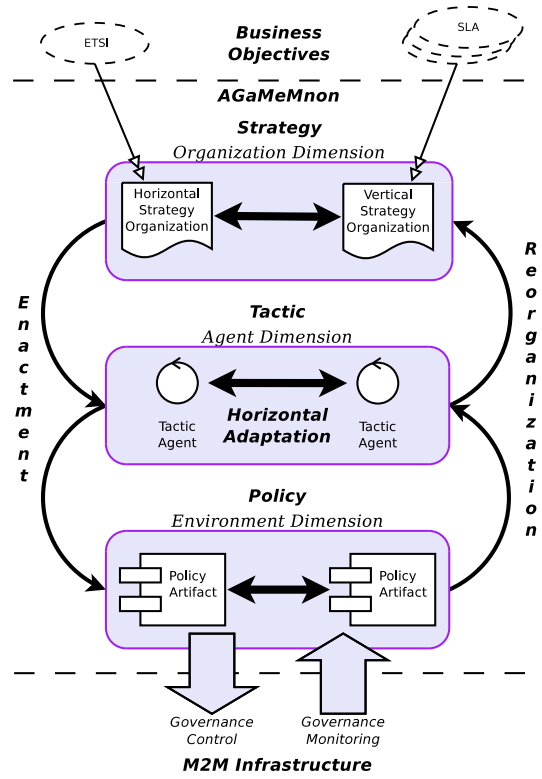




\section{Organization-based M2M Governance Strategy}

A Multi-Organizational Specification

- 1 Horizontal Organization ETSI TC M2M Functional Architecture

- Several Vertical Organizations M2M Vertical Contract

- End-to-End communication schema

- Between M2M entities

- Service Level Agreement (SLA)

$\Rightarrow$ Horizontal Flexibility

$\Leftrightarrow$ Vertical Maintenance

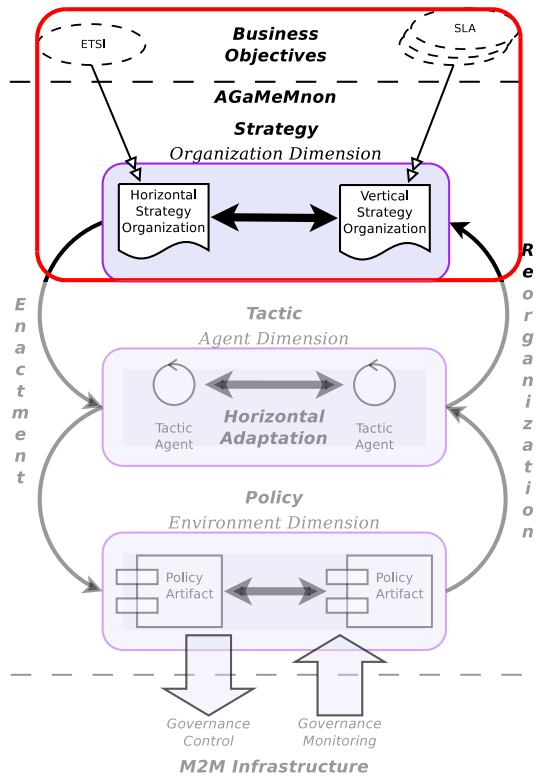




\section{Agent-based Governance Tactic}

Agent governs 1 M2M Entity : binds Horizontal and Vertical Strategy

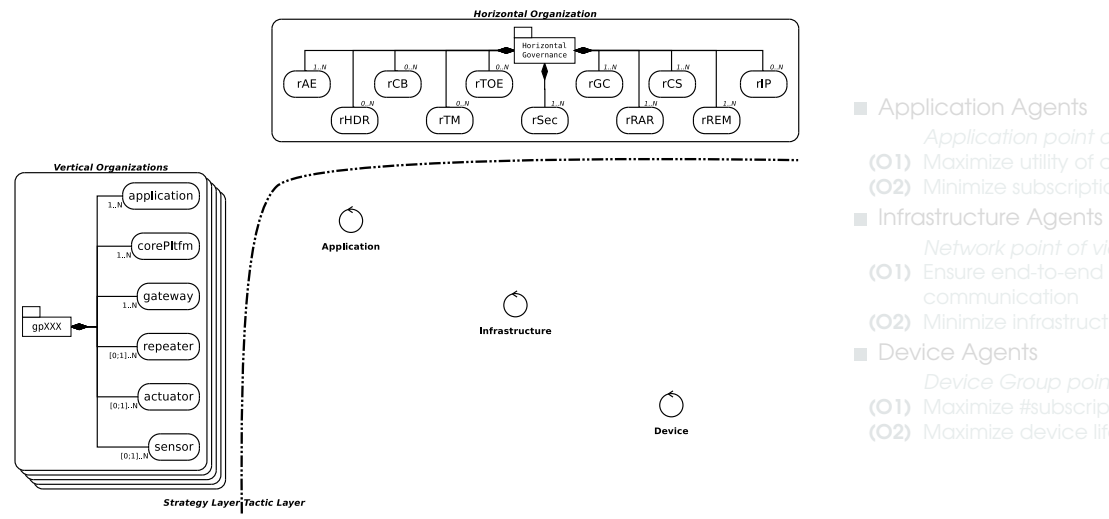




\section{Agent-based Governance Tactic}

Agent governs 1 M2M Entity : binds Horizontal and Vertical Strategy

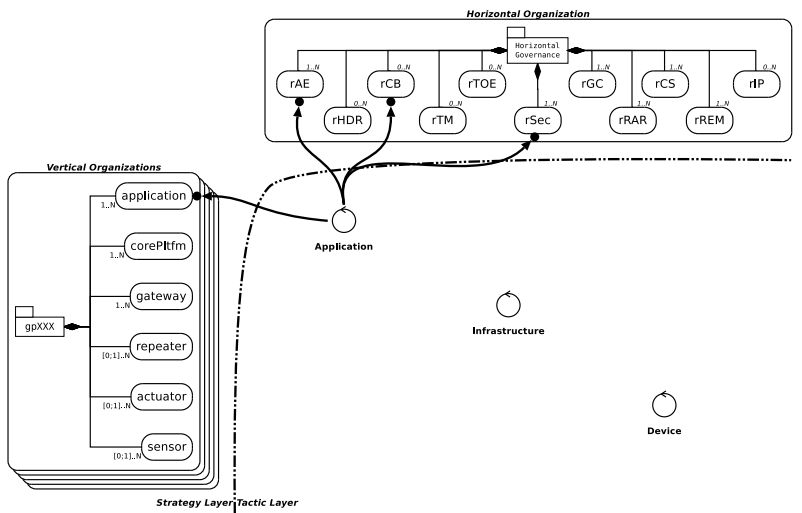

- Application Agents

Application point of view (O1) Maximize utility of contract (O2) Minimize subscription cost 


\section{Agent-based Governance Tactic}

Agent governs 1 M2M Entity : binds Horizontal and Vertical Strategy

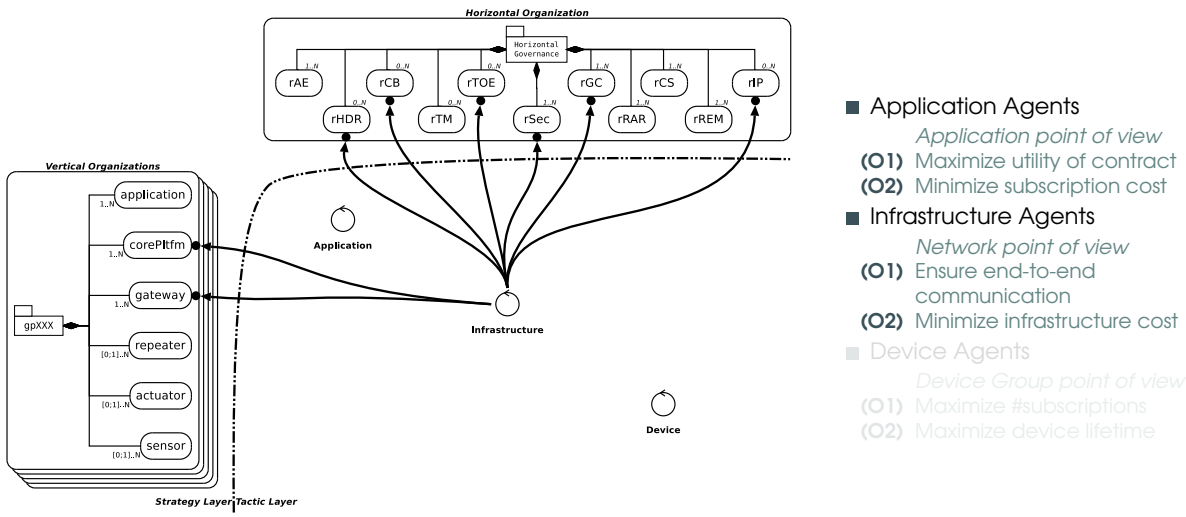




\section{Agent-based Governance Tactic}

Agent governs 1 M2M Entity : binds Horizontal and Vertical Strategy

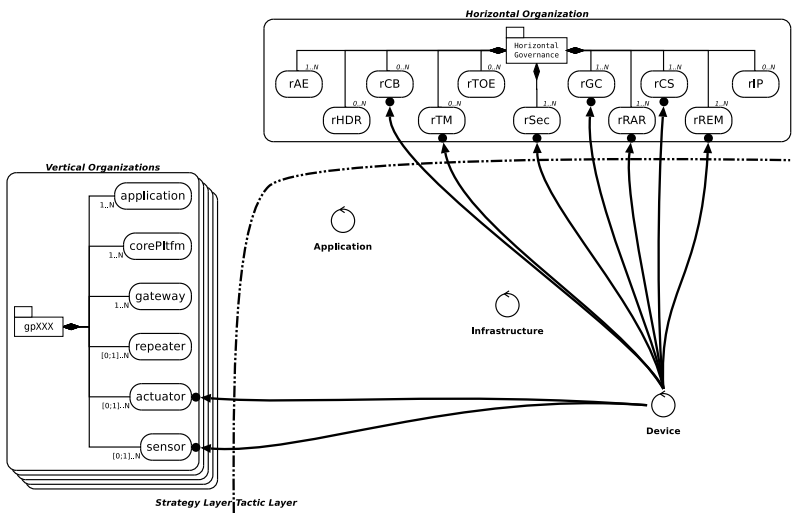

- Application Agents

Application point of view

(O1) Maximize utility of contract

(O2) Minimize subscription cost

- Infrastructure Agents

Network point of view

(O1) Ensure end-to-end communication

(O2) Minimize infrastructure cost

- Device Agents

Device Group point of view

(O1) Maximize \#subscriptions

(O2) Maximize device lifetime 


\section{Artifact-based Governance Policy}

Governance Artifact Architecture

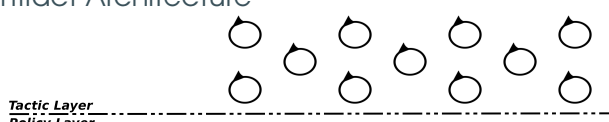

Tactic Layer

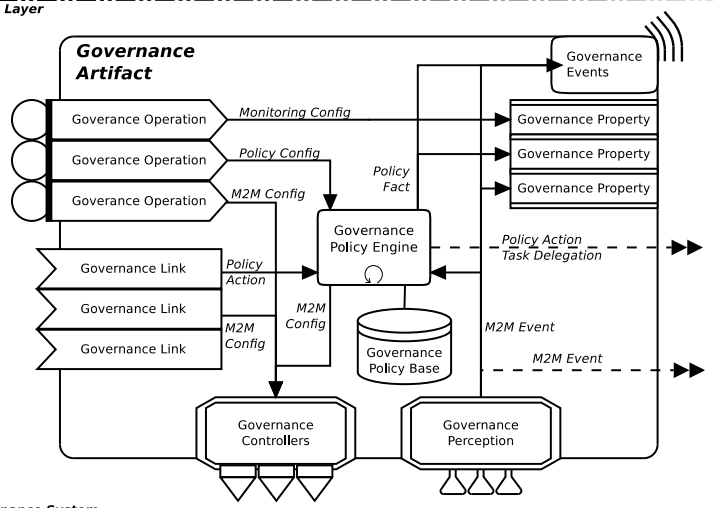

Governance System

$\overline{M 2} \bar{M}$ Infrastructure
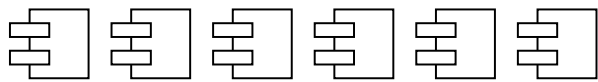


\section{Outline}

M2M, Governance and Adaptation

AGAMEMNON: an Adaptive Governance MAS for M2M

Self-reorganization in AGAMEMNON

Implementation and Evaluation 


\section{Coupling Bottom-up and Top-down Dynamics}

Organization

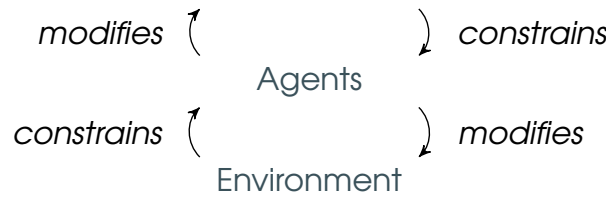




\section{Coupling Bottom-up and Top-down Dynamics}

Organization

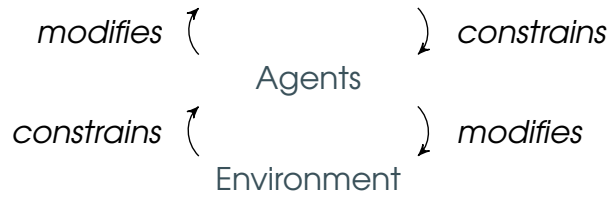

\section{Reorganization $=$}

An organization-centered adaptation process where agents are aware of the state of the organization 


\section{Coupling Bottom-up and Top-down Dynamics}

Organization

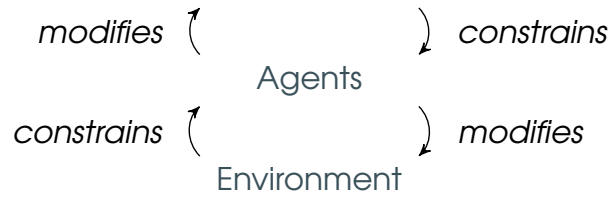

Reorganization $=$

An organization-centered adaptation process where agents are aware of the state of the organization

Self-organization =

An agent-centered process where the implicit state of the organization is the result of their activities in the environment 


\section{Coupling Bottom-up and Top-down Dynamics}

Organization

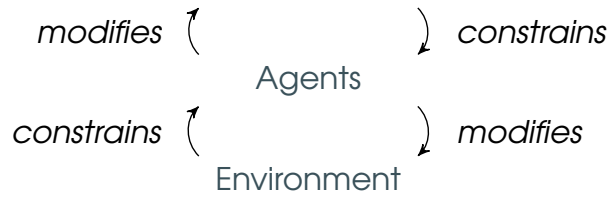

\section{Reorganization $=$}

An organization-centered adaptation process where agents are aware of the state of the organization

Self-organization =

An agent-centered process where the implicit state of the organization is the result of their activities in the environment

\section{Self-reorganization $=$}

Agents self-organize to define the reorganization process with respect to the organizational and environmental constraints 


\section{Agent-based Governance Tactic}

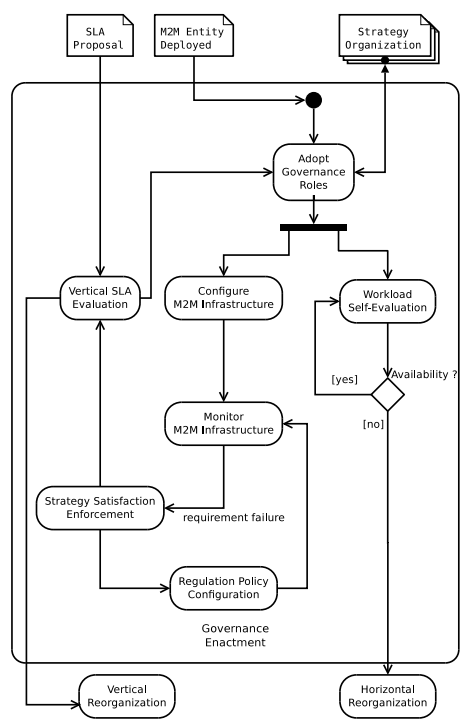

- Governance Tactic : 3 phases

- Strategy Enactment

- Horizontal Reorganization

- Vertical Reorganization

- Strategy Enactment

- Realization of strategy objectives

- M2M Infrastructure Configuration

- Enforce objectives via policies

$\Rightarrow$ Using Governance Artifact

- Horizontal/Vertical Org. Adaptation

- Adapt Governance to the M2M Infrastructure Dynamics 


\section{Adaptation by Horizontal Self-Organization}

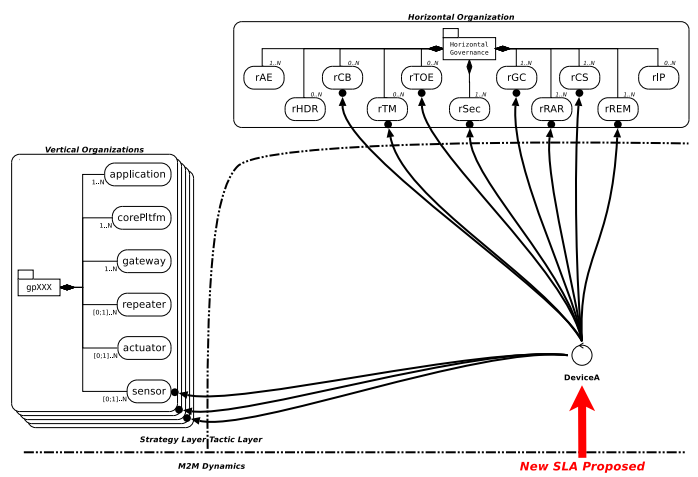

- Adapt number of agents to the governance load

- 1 Agent for 1 M2M Entity (initially)

- Overloaded Agent $\rightarrow$ Delegation

Scale-Out: Governance Coalition

- Informal Organization for 1 M2M Entity

- Each agent = 1 subset of Governance Tasks

- Coordination Heuristic before task commitment (new M2M Entity, new SLA, ...)

Scale-Down : Agent Self-termination

- Minimal task threshold

- Delegates remaining tasks

- Terminates itself 


\section{Adaptation by Horizontal Self-Organization}

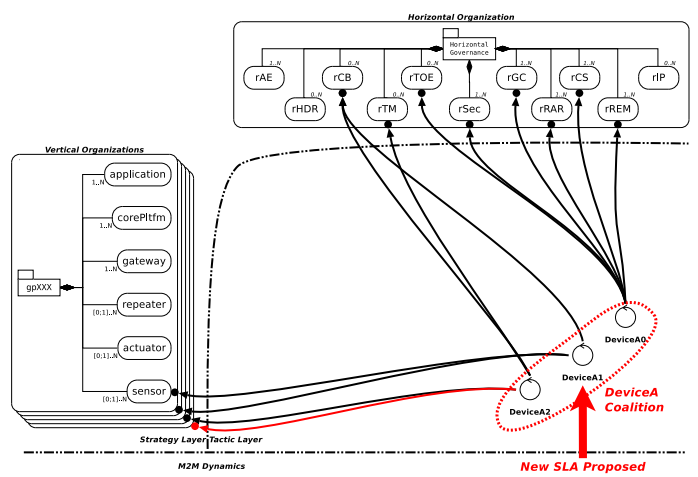

- Adapt number of agents to the governance load

- 1 Agent for 1 M2M Entity (initially)

- Overloaded Agent $\rightarrow$ Delegation

Scale-Out: Governance Coalition

- Informal Organization for 1 M2M Entity

- Each agent = 1 subset of Governance Tasks

- Coordination Heuristic before task commitment (new M2M Entity, new SLA, ...)

Scale-Down : Agent Self-termination

- Minimal task threshold

- Delegates remaining tasks

- Terminates itself 


\section{Adaptation by Vertical Reorganization}

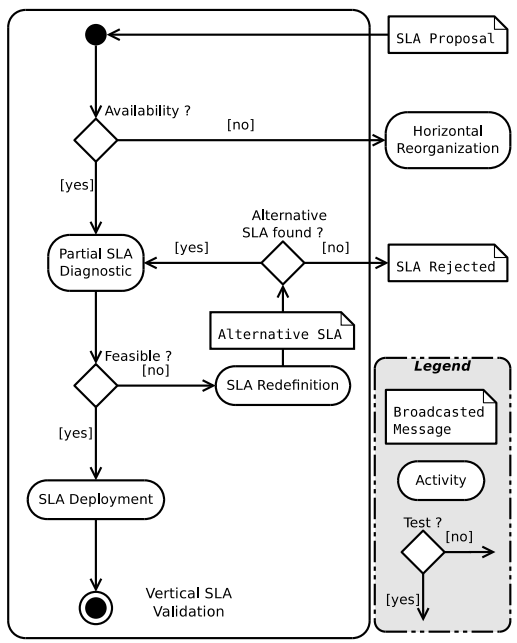

1. Handle new Vertical Contracts

- Before adopting a role in a vertical organization

- Evaluate feasibility of the SLA

- Potentially propose an Alternative SLA

2. Refine Vertical SLA to M2M Constraints

- When failing SLA

- When critical state (e.g. M2M Devices) 


\section{Outline}

\section{M2M, Governance and Adaptation}

AGAMEMnON: an Adaptive Governance MAS for M2M

Self-reorganization in AGAMEMNON

Implementation and Evaluation 


\section{Governing SensCity with AGAMEMNON}

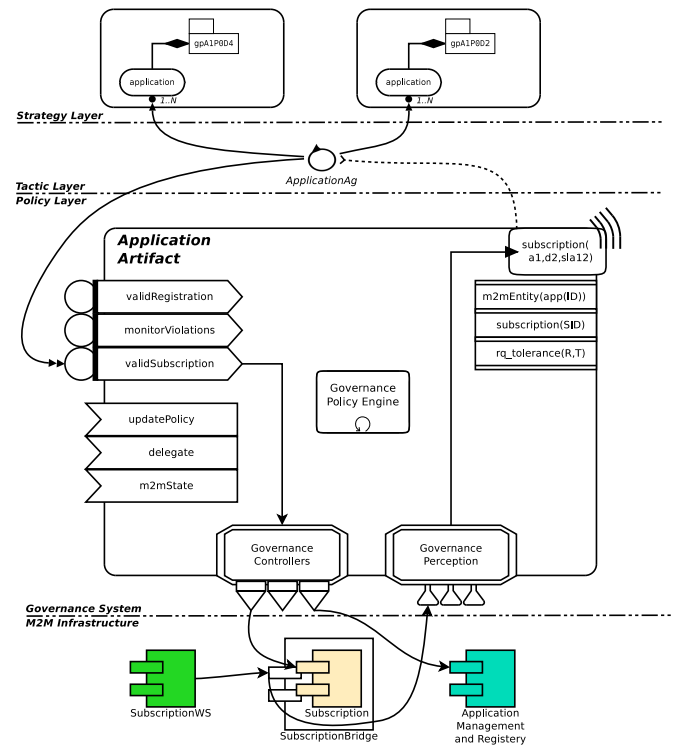




\section{Evaluation with a Simulated M2M Infrastructure}

Simulation Scenario : Smart Parking Management

- Smart Parking Scenario

- Parking Sensors in different areas

- Business Area (Peak Hour : 7AM-9AM)

- Residential Area (Peak Hour : 17PM-20PM)

- Measure Governance Impact over several (6) years

- Average accuracy of collected data

- Average devices' battery lifetime

- Different Application Requirement profiles

- ON_EVENT : Notification for each parking state change

- NOTIFy (X) : Notification of parking state each X minutes

- ADAPTIVE : Combines ON_EVENT and NOTIFY(X) profiles

$\Leftrightarrow$ Adaptation to end-user demand

- Device Battery Constraints

- Homogeneous and Affine consumption model (Scenario\#1)

- Heterogeneous and Exponential consumption model (Scenario\#2) 


\section{Evaluation with a Simulated M2M Infrastructure}

Experiment \# 1 : Application Vertical Refinement

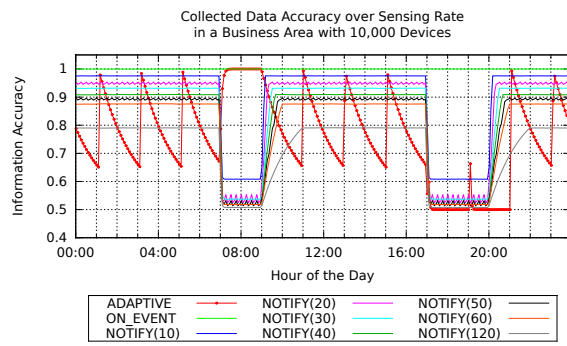

- Benefits of an explicit SLA declaration

$\Rightarrow$ Enable Governance Management

- Application point of view :

\#1 - ON_EVENT

\#2 - ADAPTIVE

$\# 3-\operatorname{NOTIFY}(X),(X \leq 20)$

- Device point of view :

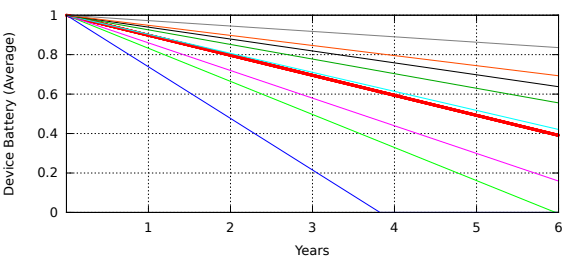

$\# 1$ - NOTIFY $(X),(X \geq 30)$

\#2 - ADAPTIVE

- ADAPTIVE profile : best trade-off between application satisfaction and device lifetime

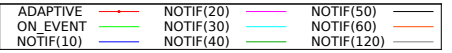




\section{Evaluation with a Simulated M2M Infrastructure}

Experiment \#2 : Device Regulation and Vertical Refinement
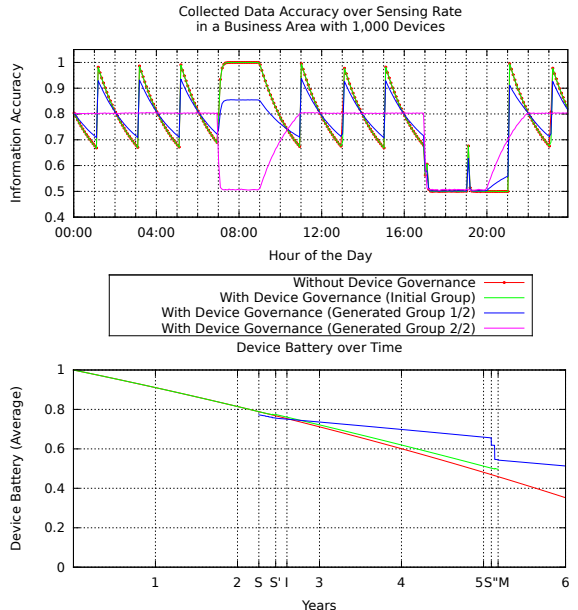
With Device Governance (Initial Group) With Device Governance (Generated Group)
- Battery consumption improvement by Governance actions :

$\Rightarrow$ Improve Average Device Lifetime

$\checkmark$ Maintain an acceptable data accuracy during 5 years

$x$ Improvement to be done after 5 years $\Rightarrow$ Finer SLA negotiation tactics 


\section{Outline}

M2M, Governance and Adaptation

AGAMEMnON: an Adaptive Governance MAS for M2M

Self-reorganization in AGAMEMNON

Implementation and Evaluation

Conclusion 


\section{Synthesis}

1. A Machine-to-Machine Governance Model

- Horizontal and Vertical concerns

- Step to horizontal M2M infrastructure deployment

- Dynamic, decentralized and adaptive governance framework

2. A Multi-Agent Oriented Programming approach for M2M Governance Adaptation and Control

- Separate governance concerns along MAS dimensions

- Multi-organizational specifications

- MAS integration within a component-based platform

- Couples an agent-centered vision and an organization-centered vision of the adaptation : self-reorganization 


\section{Synthesis}

1. A Machine-to-Machine Governance Model

- Horizontal and Vertical concerns

- Step to horizontal M2M infrastructure deployment

- Dynamic, decentralized and adaptive governance framework

2. A Multi-Agent Oriented Programming approach for M2M Governance Adaptation and Control

- Separate governance concerns along MAS dimensions

- Multi-organizational specifications

- MAS integration within a component-based platform

- Couples an agent-centered vision and an organization-centered vision of the adaptation : self-reorganization

What's next?

- Integrative framework for self-reorganization to design self-adaptive systems 

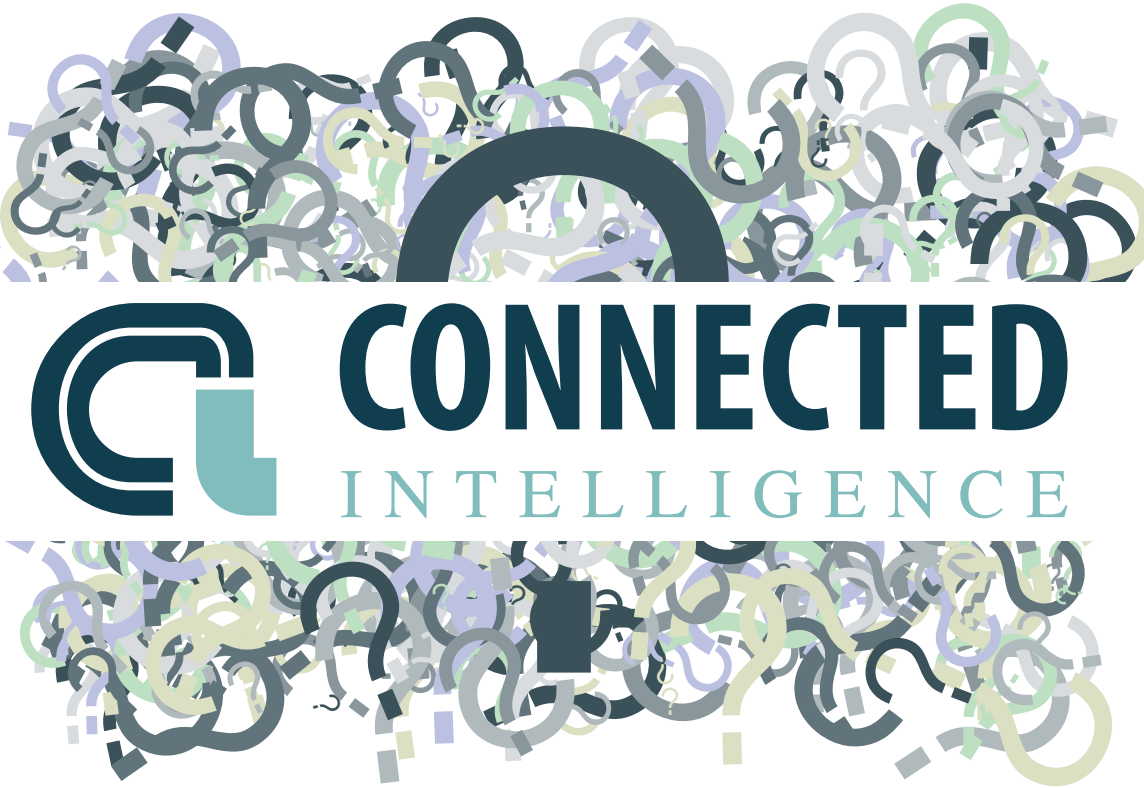

https://connected-intelligence.univ-st-etienne.fr 


\section{Bibliography (contd)}

Ian F. Akyildiz, W. Su, Y. Sankarasubramaniam, and E. Cayirci.

Wireless sensor networks : a survey.

Computer networks, 38(4):393-422, 2002.

Tim Baugé and Jesus Bernat.

Components for End to End Networking, Management and Security and Accounting.

Deliverable report WP3 / D3. 1, SENSEl, August 2008.

豆

William A. Brown and Murray Cantor.

SOA governance : how to oversee successful implementation through proven best practives and methods.

White Paper, IBM Rational Center, August 2006.

Rafael H. Bordini, Jomi F. Hübner, and Michael Wooldridge.

Programming multi-agent systems in AgentSpeak using Jason, volume 8.

Wiley-Interscience, 2008.

Dominique Barthel, Giyyarpuram Madhusudan, and Nicolas Dejean.

Application characteristics - An applicative framework for the research work conducted in ARESA2.

Deliverable Report D1. 1, ARESA2 Project, ANR 2009 Verso 017-01, September 2010. http ://aresa-project.insa-lyon.fr/. 


\section{Bibliography (contd)}

言 Yves Demazeau.

From Interactions To Collective Behaviour In Agent-Based Systems.

In Proceedings of the 7st. European Conference on Cognitive Science. Saint-Malo, pages 117-132, 1995.

ETSI,

Technical Specification 102690 V 0.14.2, Machine-to-Machine communications Functional architecture.

Technical Specification <DRAFT>, European Telecommunications Standards Institute (ETSI), November 2011.

Technical Committee Machine-to-Machine Communications (M2M).

言

R Gold, A Gluhak, N Bui, A Waller, C Sorge, F Montagut, VStirbu, H Karvonen, V Tsiatsis,

S Pennington, Z Shelby, J Vercher, M Johansson, JBohli, T Bauge, S Esfandiyari, S Haller,

E Kovacs, R Egan, F Carrez, and M Presser.

State of The Art - Sensor Frameworks and Future Internet.

Deliverable report WP3 / D3. 1, SENSEI, August 2008.

Jomi F. Hübner and Olivier Boissier.

A Model for the Structural, Functional, and Deontic Specification of Organizations in Multiagent Systems.

In Guilherme Bittencourt and Geber Ramalho, editors, Advances in Artificial Intelligence, volume 2507 of Lecture Notes in Computer Science, pages 439-448. Springer Berlin / Heidelberg, 2002. 


\section{Bibliography (contd)}

言 Tony Hayes, Max Blecher, Sushil Chatterji, Anil Jogani, John W. Lainhart IV, Rǿmulo Lomparte, Michael Schirmbrand, and Ronald Saull, editors.

COBIT 4. 1 Excerpt.

IT Governance Institute, 2007.

言 Quentin Lampin, Dominique Barthel, Isabelle Augé-Blum, and Fabrice Valois.

QoS oriented Opportunistic Routing protocol for Wireless Sensor Networks.

In Proceedings of the IFIP Wireless Days Conference 2012, Ireland, November 27-23, 2012, pages 1-6. IEEE Xplore, November 2012.

Giyyarpuram Madhusudan and André Bottaro.

City Automation, Technology for city e automation services.

White Paper, Orange Labs, Grenoble, France, 2009.

Andrea Omicini, Alessandro Ricci, and Mirko Viroli.

Artifacts in the A\&A meta-model for multi-agent systems.

Autonomous Agents and Multi-Agent Systems, 17(3) :432-456, 2008.

䎴

Michele Piunti, Olivier Boissier, Alessandro Ricci, and Jomi Fred Hübner.

Embodied Organizations : a unifying perspective in programming Agents, Organizations and Environments.

In Olivier Boissier, Amal El Fallah-Seghrouchni, Salima Hassas, and Nicolas Maudet, editors, Proceedings of The Multi-Agent Logics, Languages, and Organisations Federated Workshops (MALLOW 2010), volume 627 of CEUR Workshop Proceedings, pages 66-83, Lyon, France, August 2010. CEUR-WS.org. 


\section{Bibliography (contd)}

Z. Shelby.

Embedded Web Services.

Wireless Communications, IEEE, 17(6):52-57, 2010.

Patrick Spiess, Stamatis Karnouskos, Dominique Guinard, Domnic Savio, Olivier Baecker, Luciana Moreira Sá Souza, and Vlad Trifa.

SOA-based Integration of the Internet of Things in Enterprise Services.

In Web Services, 2009. ICWS 2009. IEEE International Conference on, pages 968-975. IEEE, 2009 .

Wefer, Gerold, editor.

Cyberinfrastructure for the US Ocean Observatories Initiative : Enabling interactive observation in the ocean. IEEE Xplore, 2009. 